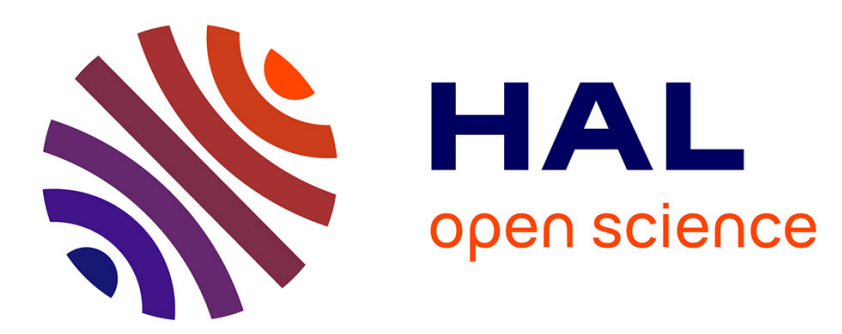

\title{
Delayed two-streams division, a diversity technique to improve signal transmission in relatively fast flat fading channels
}

Mohammad-Ali Khalighi, Laurent Ros

\section{- To cite this version:}

Mohammad-Ali Khalighi, Laurent Ros. Delayed two-streams division, a diversity technique to improve signal transmission in relatively fast flat fading channels. Signal Processing, 2005, 85, pp.705-715. hal-00078446

\section{HAL Id: hal-00078446 \\ https://hal.science/hal-00078446}

Submitted on 6 Jun 2006

HAL is a multi-disciplinary open access archive for the deposit and dissemination of scientific research documents, whether they are published or not. The documents may come from teaching and research institutions in France or abroad, or from public or private research centers.
L'archive ouverte pluridisciplinaire HAL, est destinée au dépôt et à la diffusion de documents scientifiques de niveau recherche, publiés ou non, émanant des établissements d'enseignement et de recherche français ou étrangers, des laboratoires publics ou privés. 


\title{
Delayed Two-Streams Division, A Diversity Technique To Improve Signal Transmission In Relatively Fast Flat Fading Channels *
}

\author{
Mohammad Ali Khalighi ${ }^{a}$, Laurent $\operatorname{Ros}^{b, \dagger}$ \\ ${ }^{a}$ Institut d'Électronique et de Télécommunications de Rennes (IETR), France \\ ${ }^{b}$ Laboratoire des Images et des Signaux (LIS) de Grenoble, BP46, 38402 Saint-Martin d'Hères, France
}

Received 17 November 2003; received in revised form 01 September 2004

\begin{abstract}
We consider in this paper a combination of data symbols that serves to provide time diversity and to reduce flat fading effect at receiver, when no other source of diversity is available. This technique, called delayed two-streams division (D2SD), is particularly interesting in relatively fast fading channels. By D2SD, the stream of the data symbols is divided into two substreams, the symbols of which are mixed pairwise together, and transmitted through the channel by a sufficiently long time delay between each pair. At receiver, a simple detector based on maximum likelihood criterion is used to "equalize" the symbol combination made at transmitter. The presented results show that with the negligible complexity added to the system, and while implying no loss in the spectral efficiency nor any increase in the transmit power, D2SD permits to obtain a considerable improvement in the receiver performance. Selection of the design parameters of D2SD is discussed for different cases of fading statistics, based on the bit error probability criterion.
\end{abstract}

Keywords: Fading channels, Rayleigh fading, Ricean fading, convolutive precoding, modulation diversity, codedivision multiple-access (CDMA)

\section{Introduction}

Fading mitigation in wireless channels has been one of the most challenging issues in recent years. To prevent the degradation of the signal transmission quality due to time-varying multipath propagation, diversity techniques are usually employed $[1,2]$. When, due to the limitation of cost or size, mitigation techniques based on space or polarization diversity cannot be used, the only solution may be to take use

${ }^{*}$ Parts of this work have been presented in International Symposium on Signal Processing and its Applications (ISSPA), June 2003, Paris, France (Reference [25]).

${ }^{\dagger}$ corresponding author. E-mail address: laurent.ros@lis.inpg.fr 
of time-diversity-based techniques. Among them, we can mention channel coding, convolutive precoding, and modulation diversity techniques. Convolutive precoders induce an artificial channel delay dispersion by spreading the (equivalent) channel impulse response, and average over the fading process $[3,4,5,6]$. Modulation diversity techniques (also called constellation precoding or signal space diversity) take advantage of channel time selectivity by transforming the signal constellation $[7,8,9,10,11]$.

To effectively reduce fading effect when using channel coding, we have to use low-rate codes with interleaving, or to use concatenated codes that require complex decoding [12]. Techniques like trellis coded modulation [13] also need complex decoding. On the other hand, convolutive precoders impose long delays in signal transmission $[4,5]$ and require a complex equalization of the equivalent channel $[3,4,5]$. Also, when channel is quasi-static, the complexity of the receiver remains the same. Modulation diversity techniques, in turn, need complex demodulation, while increasing the size of channel alphabet [14].

In this paper, we propose a technique, named delayed two-streams division (D2SD), permitting to reduce considerably the fading effect. It can in fact be considered as a kind of modulation diversity with a diversity order of $L=2$. It consists of a particular combination of data symbols implying no loss in spectral efficiency, and is of special interest in relatively fast fading channels. The idea of D2SD was taken from code division multiple access (CDMA) $[15,16]$ and a previous work on the subject [3]. The important contributions of our work are notably providing a simple structure for the optimal maximum likelihood (ML) detector, as well as providing tight upper and lower bounds on the corresponding bit error probability. Also, we study the performance improvement for different cases of fading statistics.

The paper is organized as follows. We present in Section 2 the D2SD combination scheme. Then, we provide in Section 3 the detector structure and expressions for the upper and lower bounds on the error probability. Performance analysis of the proposed method is performed in Section 4 for some particular channel realizations, as well as for the cases of Rayleigh and Ricean fading channels. Finally, some conclusions and discussions conclude the paper. We consider single-user communication and BPSK modulation throughout the paper. Also, we consider the conditions of flat fading where we do not dispose of the source of delay diversity that we have in frequency selective channels $[15,17,18]$. We assume that the communication channel is perfectly known at receiver.

\section{Delayed two-streams division (D2SD)}

Consider the stream of uncorrelated BPSK symbols $a \in\{+1,-1\}$ with the symbol duration $T_{b}$. It is first split into two half-rate streams $S_{1}$ and $S_{2}$ of source symbols with the duration $T_{s}=2 T_{b}$. Let us 


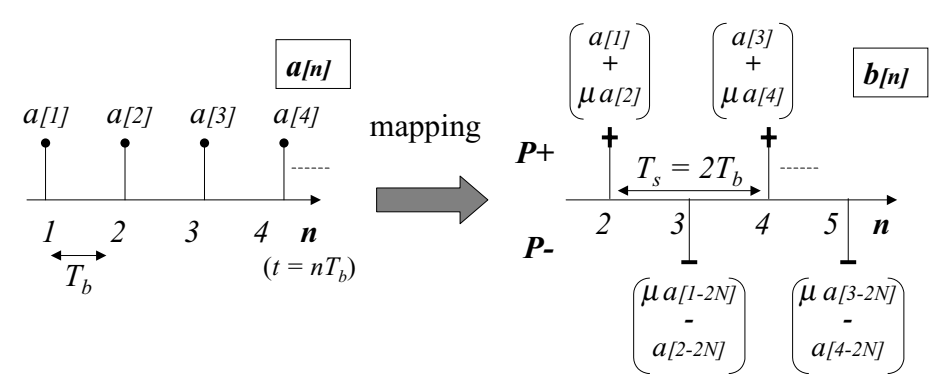

Figure 1: D2SD: combination of source symbols and division in two phases with insertion of the delay $(2 N+1) T_{b}$

denote the symbols corresponding to $S_{1}$ and $S_{2}$ by $a_{1}$ and $a_{2}$, respectively. We have:

$$
a_{1}[m]=a[2 m-1] \quad, \quad a_{2}[m]=a[2 m] \quad ; \quad m=1,2, \ldots
$$

Next, the symbols of $S_{1}$ and $S_{2}$ are combined in such a way that in a phase $P^{+}$we transmit the sum of a pair of symbols $a_{1}[m]+a_{2}[m]$, and in a phase $P^{-}$the subtraction of them, $a_{1}[m]-a_{2}[m]$. We further introduce a delay of $\left(N T_{s}+T_{b}\right)$ in the transmission of $P^{-}$relative to $P^{+}$. The combination of symbols is depicted in Fig.1. Due to a reason related to signal detection at receiver that will be explained in Section 4, we introduce further a mixture factor $\mu(0 \leq \mu \leq 1)$ in our combination. In this way, the transmit combined-symbols $b^{+}[m]$ and $b^{-}[m]$ in phases $P^{+}$and $P^{-}$will be:

$$
\left\{\begin{array}{l}
b^{+}[m] \triangleq a_{1}[m]+\mu a_{2}[m] \\
b^{-}[m] \triangleq \mu a_{1}[m]-a_{2}[m]
\end{array}\right.
$$

When transmitted through the channel, the combined symbols in phases $P^{+}$and $P^{-}$will undergo different channel fades. So, the information of $a[\mathrm{n}]$, duplicated in two phases $P^{+}$and $P^{-}$, undergoes the channel fades $\alpha[\mathrm{n}]$ and $\alpha[\mathrm{n}+2 N+1]$, respectively. ${ }^{1}$ Consider the baseband transmit signal as in (3):

$$
b(t)=T_{b} \sum_{\mathrm{n}} b[\mathrm{n}] h_{e}\left(t-\mathrm{n} T_{b}\right)
$$

where $b[\mathrm{n}]$ is the sample of the transmit signal at time $\mathrm{n} T_{b}$ :

$$
b[\mathrm{n}]=\left\{\begin{array}{ll}
b^{+}[m] & \text { for } \mathrm{n}=2 m \\
b^{-}[m-N] & \text { for } \mathrm{n}=2 m+1
\end{array} \quad \mathrm{n}=1,2, \ldots\right.
$$

and $h_{e}(\tau)$ is the half-Nyquist filter at transmitter, designed for a rate $1 / T_{b}$. The baseband equivalent complex received signal affected by fading and complex additive white Gaussian noise (AWGN) $n(t)$ is

$$
r(t)=\alpha(t) b(t)+n(t)
$$

\footnotetext{
${ }^{1}$ Notice the inserted delay between the corresponding combined-symbols.
} 


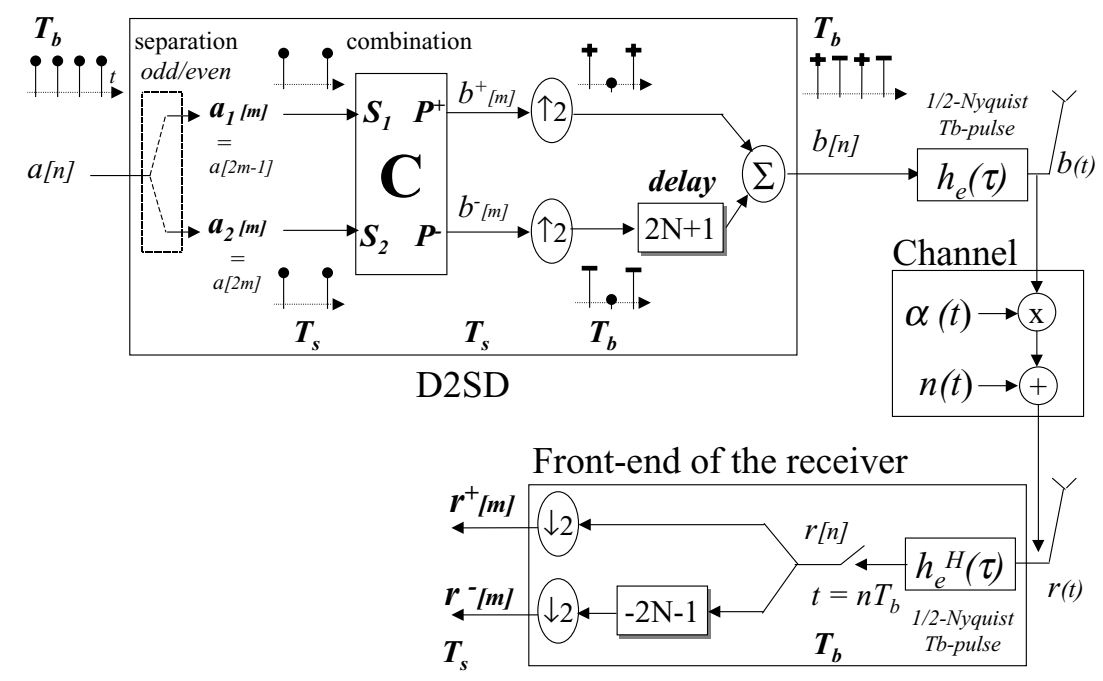

Figure 2: D2SD: symbol combination, baseband model of transmission, and the receiver front-end.

After the receiver half-Nyquist filter and synchronized sampling at time instants $\mathrm{n} T_{b}$, we obtain:

$$
r[\mathrm{n}]=\alpha[\mathrm{n}] b[\mathrm{n}]+n[\mathrm{n}]
$$

where $n[\mathrm{n}]$ is complex AWGN with the variance $\sigma^{2}$. The block diagram of Fig.2 illustrates the D2SD transmission scheme including the combination of source symbols, the baseband representation of transmitted signal, and the receiver front-end (before signal detection).

Let us now use the polyphase representation of the received signal. In accordance with our previous notations, we use superscripts . ${ }^{+}$and.$^{-}$to distinguish between two phases. So, samples of the received signals corresponding to the combined-symbols $b^{+}[m]$ and $b^{-}[m]$ will be ${ }^{2}$

$$
\left\{\begin{array}{ccc}
r^{+}[m] \triangleq & r[\mathrm{n}=2 m] & =\alpha^{+}[m] b^{+}[m]+n^{+}[m] \\
r^{-}[m] \triangleq & r[\mathrm{n}=2 m+2 N+1] & =\alpha^{-}[m] b^{-}[m]+n^{-}[m]
\end{array}\right.
$$

where $\alpha^{+}[m]=\alpha[2 m]$ and $\alpha^{-}[m]=\alpha[2 m+2 N+1]$. Also, $n^{+}$and $n^{-}$are the noise samples in two phases. The block diagram of Fig.3 shows the polyphase representation of D2SD transmission. To simplify further our notations, hereafter, we will not specify the (symbol) time index $[m]$. Assuming the conditions of relatively fast fading, with a reasonable value of $N$ (regarding the required delay in signal transmission), $\alpha^{+}$and $\alpha^{-}$will be independent random variables. Defining the vectors $\underline{r}=\left[\begin{array}{l}r^{+} \\ r^{-}\end{array}\right]$,

\footnotetext{
${ }^{2}$ Notice that although we introduced a time delay of $(2 N+1) T_{b}$ between the corresponding combinations of each pair of source symbols, the detection problem at receiver becomes in fact that of an instantaneous mixture of them while imposing a delay in signal detection (the negative delay shown on Fig.2 and 3).
} 


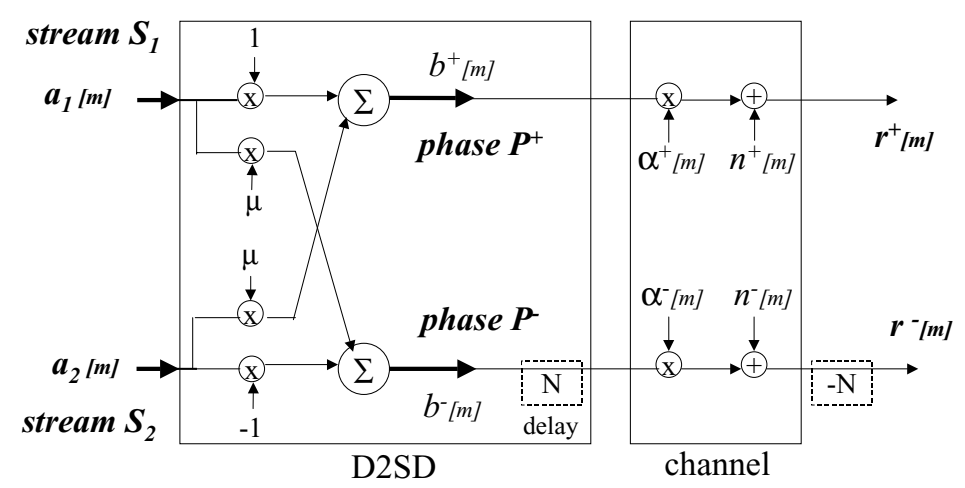

Figure 3: Polyphase representation of D2SD transmission

$$
\begin{aligned}
\underline{b}=\left[\begin{array}{c}
b^{+} \\
b^{-}
\end{array}\right], \text {and } \underline{a}=\left[\begin{array}{c}
a_{1} \\
a_{2}
\end{array}\right], \text { and using }(7) \text { and }(2), \text { we can write: } \\
\qquad \underline{r}=\boldsymbol{G}\left[\begin{array}{l}
a_{1} \\
a_{2}
\end{array}\right]+\left[\begin{array}{c}
n^{+} \\
n^{-}
\end{array}\right] \text {with: } \boldsymbol{G}=\left[\begin{array}{cc}
\alpha^{+} & \mu \alpha^{+} \\
\mu \alpha^{-} & -\alpha^{-}
\end{array}\right]
\end{aligned}
$$

We further define the matrix $C$ as follows.

$$
\boldsymbol{C}=\left[\begin{array}{cc}
1 & \mu \\
\mu & -1
\end{array}\right] \quad ; \quad \boldsymbol{G}=\left[\begin{array}{cc}
\alpha^{+} & 0 \\
0 & \alpha^{-}
\end{array}\right] \boldsymbol{C}
$$

There is a tight analogy between the D2SD and CDMA signaling [16]: we can consider our transmission scheme like the combination of the symbols of two users in a multiple access channel. In this way, the symbols in streams $S_{1}$ and $S_{2}$ belong to users \#1 and \#2, respectively. The phases $P^{+}$and $P^{-}$can hence be regarded as chips \#1 and \#2. However, in contrast to CDMA, here we introduced a delay of $(2 N+1) T_{b}$ in the transmission of $P^{+}$and $P^{-}$, and also introduced the mixture factor $\mu$. By this analogy, $\boldsymbol{C}$ can in fact be regarded as a matrix of non-binary orthogonal $\operatorname{codes}^{3}$, whereas $\boldsymbol{G}$ can be seen as the matrix of non-orthogonal codes due to the fading effect, although we have flat fading conditions. Hence, at receiver, our problem is similar to that of multi-user detection; with multiple-access interference but without inter-symbol interference.

\section{Detector structure}

For a non-fading channel, or a very slowly varying channel where the channel coherence time $\tau_{c} \gg N T_{s}$ for a pre-defined $N$, we have $\alpha^{+}=\alpha^{-}$and so, $\boldsymbol{G}$ remains orthogonal. The detection of symbols is easy in this case, and is done in an optimum manner by the matched filter (MF) $\boldsymbol{G}^{H}=\alpha^{*} \boldsymbol{C}^{T}$, where

\footnotetext{
${ }^{3}$ Concerning chips \#1 and \#2, we have unequal power for each user code, but equal power for the ensemble of users.
} 
superscripts $.^{T}, .^{H}$ and.$^{*}$ denote transpose, transpose-conjugate, and complex conjugate, respectively. In other words, D2SD is transparent to non-fading channels.

Let us consider the general case of fading channel, i.e., $N T_{s} \geq \tau_{c}$. We assume that the channel is estimated perfectly at receiver, and hence, $\alpha^{+}$and $\alpha^{-}$are known exactly. We choose the optimum ML detector to detect $a_{1}$ and $a_{2}$ from the received signal $\underline{r}$ and propose a very simple implementation for it. As we will see later in Subsection 3.1, the ML detection is done at the output of the MF to "channel+code", $\boldsymbol{G}^{H}$, which gives the sampled signal at rate $1 / T_{s}$ on the recovered streams $S_{1}$ and $S_{2}$ :

$$
\underline{y}=\left[\begin{array}{l}
y_{1} \\
y_{2}
\end{array}\right]=\boldsymbol{G}^{H}\left[\begin{array}{l}
r^{+} \\
r^{-}
\end{array}\right]=\boldsymbol{\Gamma}\left[\begin{array}{l}
a_{1} \\
a_{2}
\end{array}\right]+\left[\begin{array}{c}
n_{1}^{\prime} \\
n_{2}^{\prime}
\end{array}\right]
$$

where,

$$
\boldsymbol{G}^{H}=\left[\begin{array}{cc}
\alpha^{+*} & \mu \alpha^{-*} \\
\mu \alpha^{+^{*}} & -\alpha^{+^{*}}
\end{array}\right] \quad ; \quad \boldsymbol{\Gamma} \triangleq \boldsymbol{G}^{H} \boldsymbol{G}=\left[\begin{array}{cc}
\left|\alpha^{+}\right|^{2}+\mu^{2}\left|\alpha^{-}\right|^{2} & \mu\left(\left|\alpha^{+}\right|^{2}-\left|\alpha^{-}\right|^{2}\right) \\
\mu\left(\left|\alpha^{+}\right|^{2}-\left|\alpha^{-}\right|^{2}\right) & \mu^{2}\left|\alpha^{+}\right|^{2}+\left|\alpha^{-}\right|^{2}
\end{array}\right] .
$$

$n^{\prime}[$.$] is the noise sample at the MF output and |.| denotes modulus.$

\subsection{Maximum likelihood detection of data symbols}

Let $\hat{a}_{1}$ and $\hat{a}_{2}$ be the hard decisions made by the ML detector on the transmitted symbols $a_{1}$ and $a_{2}$. It is straight forward to show that for jointly ML detection of $\left(a_{1}, a_{2}\right)$, we have to look for $\left(\hat{a}_{1}, \hat{a}_{2}\right)$ that minimize the following expression. ${ }^{4}$

$$
\omega=\left|r^{+}-\left(\alpha^{+} \hat{a}_{1}+\mu \alpha^{+} \hat{a}_{2}\right)\right|^{2}+\left|r^{-}-\left(\mu \alpha^{-} \hat{a}_{1}-\alpha^{-} \hat{a}_{2}\right)\right|^{2}
$$

After some manipulations, while neglecting constant terms that do not depend on $\hat{a}_{1}$ nor on $\hat{a}_{2}$, the ML detection reduces to the maximization of the function $\Omega$ :

$$
\Omega=\hat{a}_{1} \Re\left\{y_{1}\right\}+\hat{a}_{2} \Re\left\{y_{2}\right\}-\hat{a}_{1} \hat{a}_{2} \mu\left(\left|\alpha^{+}\right|^{2}-\left|\alpha^{-}\right|^{2}\right)
$$

$\Re\{$.$\} denotes the real part operator. Let us define$

$$
\rho^{\prime}=\mu\left(\left|\alpha^{+}\right|^{2}-\left|\alpha^{-}\right|^{2}\right) .
$$

It can be shown that the detector outputs, or in other words, the jointly optimal hard decisions on $\left(a_{1}, a_{2}\right)$, are obtained from (15), in which $\operatorname{sgn}($.$) is the sign function and |.| the absolute value [20].$

$$
\left\{\begin{array}{l}
\hat{a}_{1}=\operatorname{sgn}\left(\Re\left\{y_{1}\right\}+\frac{1}{2}\left|\Re\left\{y_{2}\right\}-\rho^{\prime}\right|-\frac{1}{2}\left|\Re\left\{y_{2}\right\}+\rho^{\prime}\right|\right) \\
\hat{a}_{2}=\operatorname{sgn}\left(\Re\left\{y_{2}\right\}+\frac{1}{2}\left|\Re\left\{y_{1}\right\}-\rho^{\prime}\right|-\frac{1}{2}\left|\Re\left\{y_{1}\right\}+\rho^{\prime}\right|\right)
\end{array}\right.
$$

Notice that $\left|\Omega\left(\hat{a}_{1}, \hat{a}_{2}\right)\right|$ can be considered as a measure of reliability of the decision in (15).

\footnotetext{
${ }^{4}$ Here the ML detection is equivalent to GLRT (Generalized Likelihood Ratio Test) detection [19].
} 


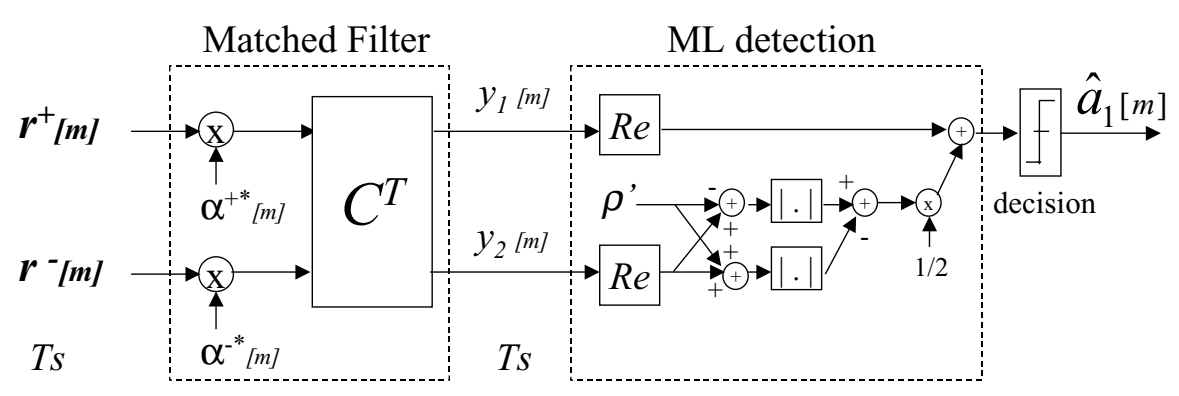

Figure 4: ML detector giving hard decisions on the transmitted (source) symbols of stream $S_{1}\left(a_{1}\right)$.

\subsection{Error probability}

A closed form expression for the exact error probability $P_{e}$ can not be obtained. We provide, instead, expressions for an upper and a lower bound on $P_{e}$. For a given pair of source symbols $\left(a_{1}, a_{2}\right)$, the error probability on each one will be different, depending on the channel gains $\left(\alpha^{+}, \alpha^{-}\right)$corresponding to the combined symbols $\left(b^{+}, b^{-}\right)$. Let us denote the error probabilities on $a_{1}$ and $a_{2}$ by $P_{e, a_{1}}$ and $P_{e, a_{2}}$, respectively, resulting from the joint ML detection of this pair of symbols. Inspiring by the approach of Verdù in the case of multiuser detection [16], we provide in the following, bounds on these probabilities as a function of $\left(\alpha^{+}, \alpha^{-}\right)[20]$. Let us first define the following probabilities:

$$
\begin{gathered}
P_{1, a_{1}}^{\prime}=\frac{1}{2} \operatorname{erfc}\left(\sqrt{\frac{\left|\alpha^{+}\right|^{2}+\mu^{2}\left|\alpha^{-}\right|^{2}}{\sigma^{2}}}\right) \\
P_{2, a_{1}}^{\prime}=\frac{1}{2} \operatorname{erfc}\left(\sqrt{\frac{\left|\alpha^{+}\right|^{2}(\mu+1)^{2}+\left|\alpha^{-}\right|^{2}(\mu-1)^{2}}{\sigma^{2}}}\right) \\
P_{3, a_{1}}^{\prime}=\frac{1}{2} \operatorname{erfc}\left(\sqrt{\frac{\left|\alpha^{+}\right|^{2}(\mu-1)^{2}+\left|\alpha^{-}\right|^{2}(\mu+1)^{2}}{\sigma^{2}}}\right)
\end{gathered}
$$

where $\operatorname{erfc}(x)=\frac{2}{\sqrt{\pi}} \int_{x}^{\infty} e^{-t^{2}} d t$, and $\sigma^{2}=2 N_{0} / T_{b}$.

The upper and lower bounds on $P_{e, a_{1}}$, denoted by $P_{\text {upper }, a_{1}}$ and $P_{\text {lower }, a_{1}}$, are:

$$
P_{\text {upper }, a_{1}}=P_{1, a_{1}}^{\prime}+\frac{P_{2, a_{1}}^{\prime}}{2}+\frac{P_{3, a_{1}}^{\prime}}{2} \quad ; \quad P_{\text {lower }, a_{1}}=\max \left\{P_{1, a_{1}}^{\prime}, \frac{P_{2, a_{1}}^{\prime}}{2}, \frac{P_{3, a_{1}}^{\prime}}{2}\right\}
$$

By interchanging $\alpha^{+}$and $\alpha^{-}$in (16), (17), (18) we obtain $P_{1, a_{2}}^{\prime}, P_{2, a_{2}}^{\prime}$, and $P_{3, a_{2}}^{\prime}$, the corresponding probabilities for $a_{2}$. Meanwhile, we notice that $P_{2, a_{2}}^{\prime}=P_{3, a_{1}}^{\prime}$ and $P_{3, a_{2}}^{\prime}=P_{2, a_{1}}^{\prime}$. The upper and lower bounds on $P_{e, a_{2}}$ are then:

$$
P_{\text {upper }, a_{2}}=P_{1, a_{2}}^{\prime}+\frac{P_{2, a_{2}}^{\prime}}{2}+\frac{P_{3, a_{2}}^{\prime}}{2} \quad ; \quad P_{\text {lower }, a_{2}}=\max \left\{P_{1, a_{2}}^{\prime}, \frac{P_{2, a_{2}}^{\prime}}{2}, \frac{P_{3, a_{2}}^{\prime}}{2}\right\}
$$

The error probability $P_{e}$, averaged on a pair of $\left(a_{1}, a_{2}\right)$ is:

$$
P_{e}=\frac{P_{e, a_{1}}+P_{e, a_{2}}}{2}
$$


and the upper and lower bounds $P_{\text {upper }}$ and $P_{\text {lower }}$ on $P_{e}$ are as follows.

$$
P_{\text {upper }}=\frac{P_{\text {upper }, a_{1}}+P_{\text {upper }, a_{2}}}{2} \quad, \quad P_{\text {lower }}=\frac{P_{\text {lower }, a_{1}}+P_{\text {lower }, a_{2}}}{2}
$$

We will see that these bounds are tight enough and quite useful in studying the receiver performance.

\section{Performance analysis of D2SD}

To study the performance of D2SD, we first consider some particular channel realizations in Subsection 4.1, before treating the cases of Rayleigh and Ricean fading in Subsections 4.3 and 4.4, respectively.

\subsection{Particular channel realizations}

\subsubsection{Performance evaluation}

We provide performance curves in terms of the error probability $P_{e}$ versus $E_{b} / N_{0}$ for a given channel realization, that is, for a particular pair of channel gains $\alpha^{+}$and $\alpha^{-}$. From (8) the "instantaneous" received energy per $T_{b}$ includes a part relating to $a_{1}$ and the other part relating to $a_{2}$. We separate the energies corresponding to $a_{1}$ and $a_{2}$, that we call $E_{b 1}$ and $E_{b 2}$, respectively:

$$
E_{b 1}=\frac{T_{b}}{2}\left(\left|\alpha^{+}\right|^{2}+\mu^{2}\left|\alpha^{-}\right|^{2}\right) \quad, \quad E_{b 2}=\frac{T_{b}}{2}\left(\mu^{2}\left|\alpha^{+}\right|^{2}+\left|\alpha^{-}\right|^{2}\right) .
$$

The local average received energy (over $a_{1}$ and $a_{2}$ symbols) per $T_{b}$ is then:

$$
E_{b}=\frac{1}{2}\left(E_{b 1}+E_{b 2}\right)=\frac{T_{b}}{4}\left(\left|\alpha^{+}\right|^{2}+\left|\alpha^{-}\right|^{2}\right)\left(1+\mu^{2}\right)
$$

and the local average error probability $P_{e}$ is given by (21).

\subsubsection{Impact of the mixture factor $\mu$}

The mixture factor $\mu$ has an important impact on the receiver performance and should be chosen appropriately. It can in fact be regarded as a "degree of freedom" that we do not dispose in binary CDMA coding, for example. Here, a trade off should be considered in the choice of $\mu$ : The closer $\mu$ is to 1 , the better we can profit in terms of fading reduction, but the interference of the other symbol will be more important too (interference of $a_{2}$ in the detection of $a_{1}$, for example).

Let us define the factor $f=\frac{\alpha^{+}}{\alpha^{-}}$. We want to see the effect of $f$ on the error probability for a given $\mu$. Notice that $f$ close to 1 signifies a small change in the channel gain from the time reference $T_{b}$ to $(2 N+1) T_{b}$, whereas a large $f$ signifies a deep channel fade in the latter reference time. Fig.5 shows 


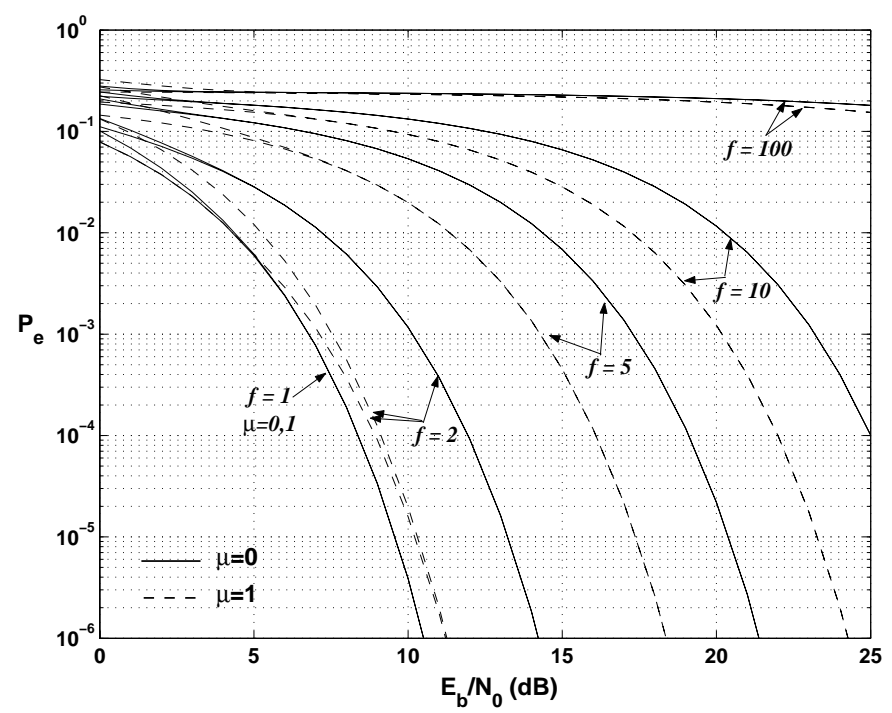

(a)

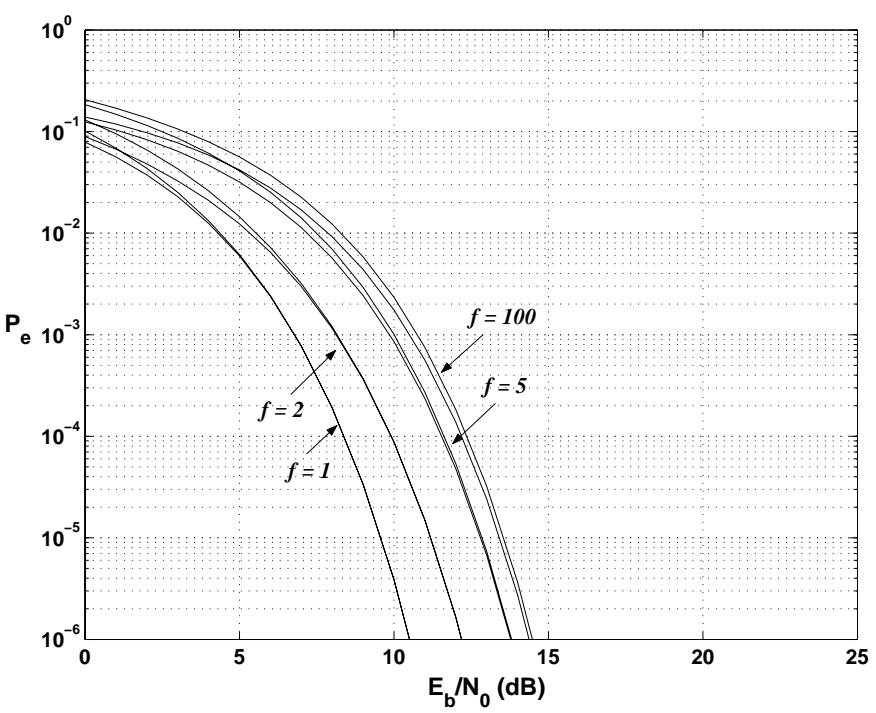

(b)

Figure 5: Impact of $\mu$ on D2SD performance, particular channel realization with $f=\frac{\alpha^{+}}{\alpha^{-}} ;$(a) $\mu=0,1 ;(\mathrm{b}) \mu=0.5$

curves of (upper and lower bounds on) $P_{e}$ versus $E_{b} / N_{0}$ for $\mu=0,0.5,1$ and different values of $f .^{5}$

$\mu=0$ : No mixture is performed on the symbols of $S_{1}$ and $S_{2}$ actually, and hence, we see purely the effect of the channel fading on $P_{e}{ }^{6}$ So, the worst performance is obtained for the same $f(>1)$ as compared to the two other cases. For $f \rightarrow \infty$, in high SNR we can decide correctly on $a_{1}$ (or $a_{2}$ ) only, and so, $P_{e} \rightarrow 1 / 4$.

$\underline{\mu=1}$ : We have the maximum fading reduction but the interference is maximum too. So, the performance is better than for $\mu=0$, but worse than for $\mu=0.5$ for large $f$. For $f \rightarrow \infty$, in high SNR we can decide correctly on $a_{1}$ and $a_{2}$ only when they are equal, and hence, $P_{e} \rightarrow 1 / 4$.

$\underline{\mu=0.5}$ : For large enough $f$ the performance is better than that for $\mu=0$ and $\mu=1$, and the maximum degradation with respect to the no-fading case is about 3 to $4 \mathrm{~dB}$. For $f \rightarrow \infty$ where $b^{-}$is lost, the decision on $a_{1}$ and $a_{2}$ should be performed using only $b^{+}$, where in the presence of noise the best performance is obtained for $\mu_{o p t}=0.5$.

It could be seen that for $f>1$ the performances of ZF or MMSE detectors degrade considerably as compared to ML detector, since these solutions consist in inverting (exactly or partially) the matrix $\boldsymbol{\Gamma}$.

\footnotetext{
${ }^{5}$ Notice that for $f=1$ (no fading), the performance of the system is independent of $\mu$, and the optimum decisions on $a_{1}$ and $a_{2}$ are made after the MF $\boldsymbol{G}^{H}$ simply. In this case we obtain $P_{e}=\frac{1}{2} \operatorname{erfc}\left(\sqrt{E_{b} / N_{0}}\right)$.

${ }^{6}$ The error probability for $\mu=0$ is given by $P_{e}=\left(P_{e, a_{1}}+P_{e, a_{2}}\right) / 2=\frac{1}{4} \operatorname{erfc}\left(\sqrt{\frac{E_{b 1}}{N_{0}}}\right)+\frac{1}{4} \operatorname{erfc}\left(\sqrt{\frac{E_{b 2}}{N_{0}}}\right)$.
} 


\subsection{Case of fading channel}

The performance improvement obtained by D2SD depends on the fading statistics. We expect to obtain a more important gain in the case of Rayleigh fading, as compared to the Ricean fading, since the former case undergoes more severe channel variations.

\subsubsection{Performance evaluation for a random channel}

Remember that regarding the choice of $N$, i.e. the delay imposed in signal transmission, D2SD is suitable for relatively fast fading channels. So, it is quite reasonable to consider the conditions of ergodic channel, and to consider the average error probability $\bar{P}_{e}=\mathrm{E}\left\{P_{e}\right\}$ for the performance evaluation (E\{. $\}$ denotes expected value). We should hence use the average received SNR $\bar{E}_{b} / N_{0}$ in the performance analysis, with $\bar{E}_{b}=\mathrm{E}\left\{E_{b}\right\}$ the average received energy per $T_{b}$ at the receiver input. According to our previous definitions, $\bar{E}_{b}=\left(1+\mu^{2}\right) \frac{T_{b}}{2} \overline{|\alpha|}^{2}$, where $\overline{|\alpha|}^{2}=\mathrm{E}\left\{|\alpha[\mathrm{n}]|^{2}\right\}$.

\subsection{Rayleigh fading}

\subsubsection{Error probability computation}

The upper and lower bounds on $\bar{P}_{e}$ can be computed by means of Monte Carlo simulations using the expressions given in Subsection 3.2. We can, however, obtain an approximate analytical expression on the upper bound, as explained in the following.

We have $\operatorname{erfc}(x) \leq e^{-x^{2}}$. By replacing erfc(.) function in (16), (17), (18) by $e^{-x^{2}}$ we obtain a somewhat looser but still useful upper bound, as we will show. We can now easily average the upper bound over $\alpha^{+}$and $\alpha^{-}$, from the PDF of $\rho=|\alpha|$, given by (25) for Rayleigh distribution:

$$
P(\rho)=2 \rho \exp \left(-\rho^{2}\right)
$$

After some manipulations, we obtain the following analytical expression for the new upper bound.

$$
P_{u p}^{\prime}=\frac{1}{4}\left(1+\frac{1}{\sigma^{2}}\right)^{-1}\left(1+\frac{\mu^{2}}{\sigma^{2}}\right)^{-1}+\frac{1}{4}\left(1+\frac{(1+\mu)^{2}}{\sigma^{2}}\right)^{-1}\left(1+\frac{(1-\mu)^{2}}{\sigma^{2}}\right)^{-1}
$$

We can further simplify this bound in high SNR where we obtain:

$$
P_{u p}^{\prime} \approx \frac{1}{4}\left(\frac{E_{b}}{N_{0}}\right)^{-2}\left(\mu^{-2}+\left(1-\mu^{2}\right)^{-2}\right)\left(1+\mu^{2}\right)^{2} \quad ; \quad \frac{E_{b}}{N_{0}} \gg 1
$$

\subsubsection{Choice of mixture factor $\mu$}

From (27) we obtain the optimal choice of $\mu=0.53$ minimizing $P_{u p}^{\prime}$, independently of SNR. However, remember that $(27)$ is valid for high SNR. For low SNR, $P_{u p}^{\prime}$ from $(26)$ as well as $P_{\text {upper }}$ and $P_{\text {lower }}$ 


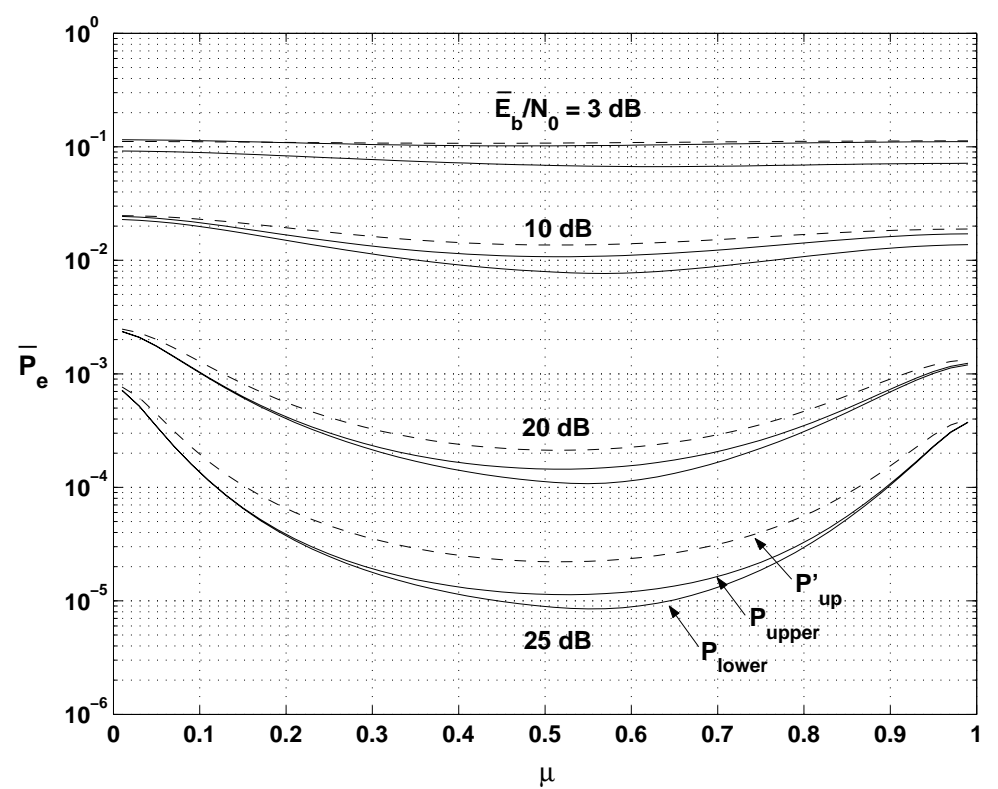

Figure 6: Effect of $\mu$ on the performance of D2SD, Rayleigh flat fading

from (22) do depend on SNR. We have shown in Fig.6 bounds on $\bar{P}_{e}$ versus $\mu$ for four values of $\bar{E}_{b} / N_{0}$. Remember the trade off in the choice of $\mu$ between the induced interference and the fading reduction. For $E_{b} / N_{0} \geq 10 \mathrm{~dB}$, the optimal choice is between 0.52 and 0.57 . In lower SNR the exact $\mu_{\text {opt }}$ depends on SNR, varying between 0.4 and 0.6 ; however, $\bar{P}_{e}$ has a poor sensitivity to $\mu$ in this interval.

We will take $\mu_{o p t}=0.55$. Notice that this $\mu_{o p t}$ is a little larger than 0.5 , which was the optimum choice for a channel with no-or-severe fading (see Subsection 4.1.2 for $f \rightarrow \infty$ ).

Let us compare our result with those in [10] and [8] for the modulation diversity with the diversity order $L=2$ under Rayleigh fading. Here, our criterion to find $\mu_{o p t}$ was to obtain the minimum $\bar{P}_{e}$. In [10] the optimization criterion for the design of the constellation transformation has been to maximize the minimum product distance between any two points of the transformed constellation. With our notations, by interchanging the columns of $\boldsymbol{C}$, we would obtain $\mu_{\text {opt }}=2 /(1+\sqrt{5})=0.618$ (see [10], Paragraph VI.A). In [8] the optimization has been in terms of the channel cut-off rate. The transformation consists of a rotation matrix with the optimum rotation angle $\phi$ of $29.63^{\circ}$ (see [8], Section V). This corresponds to a mixture factor of $\mu_{o p t}=\sin (\phi) / \cos (\phi)=0.57$. We see that in both works the proposed $\mu_{o p t}$ is very close to that we obtained for D2SD.

\subsubsection{Fading reduction; comparison with SISO and SIMO}

Taking $\mu_{o p t}=0.55$, we want to see how much we gain in terms of fading reduction. For this purpose, we compare D2SD with a simple single-antenna system (without any source of diversity) and a doublereceive antenna system using MRC (Maximal Ratio Combining) detection. We will call these two cases SISO (single-input single-output) and SIMO (single-input multiple-outputs), respectively. Considering 


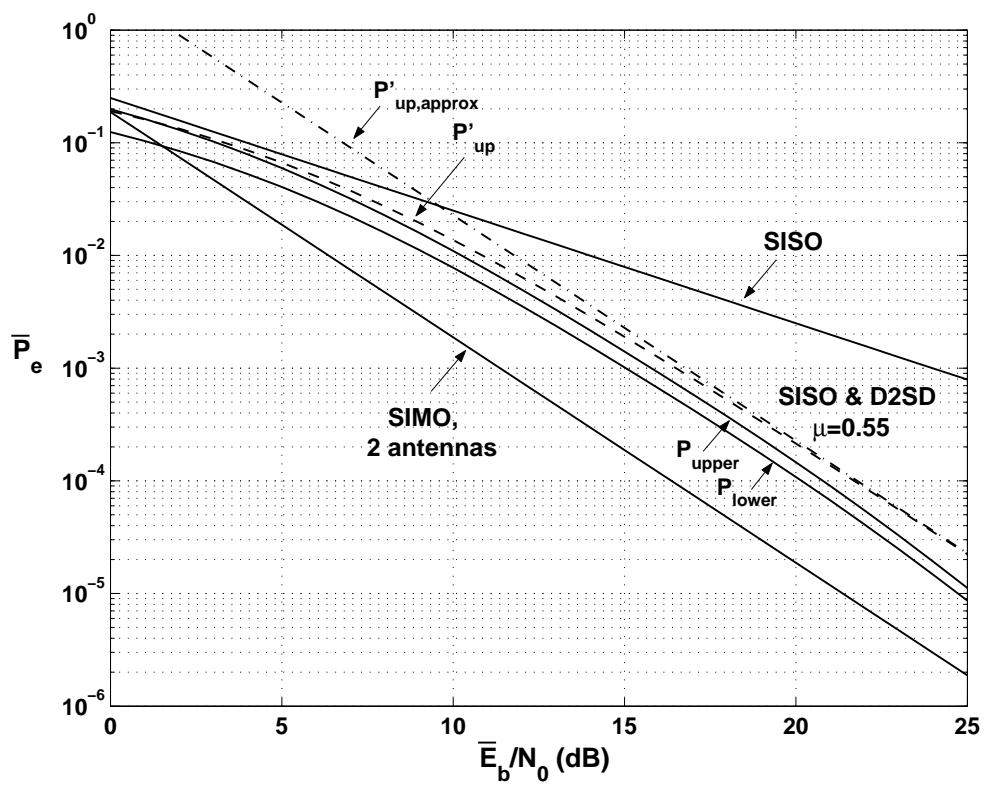

Figure 7: Performance comparison of D2SD, SISO, and SIMO, Rayleigh flat fading

Rayleigh flat fading conditions, Fig.7 contrasts the performances of D2SD, SISO, and SIMO. For D2SD, the upper and lower bounds on $\bar{P}_{e}$ are shown versus $\bar{E}_{b} / N_{0}$, whereas for the two other cases, we have shown curves of asymptotic $\bar{P}_{e}$ in high SNR, given below [2]. ${ }^{7}$

$$
\bar{P}_{e} \approx\left(\frac{1}{4 \bar{E}_{b} / N_{0}}\right)^{M_{R}}\left(\begin{array}{c}
2 M_{R}-1 \\
M_{R}
\end{array}\right)
$$

$M_{R}=1$ for SISO and $M_{R}=2$ for SIMO and $\left(\begin{array}{c}P \\ Q\end{array}\right)=\frac{P !}{Q !(P-Q) !}$. Notice that no coding is used for either system. From Fig.7 we see that our system outperforms the simple SISO in high SNR. It has a degradation of about $3 \mathrm{~dB}$, as compared to the SIMO system. This is, in fact, the very reasonable price paid for the mixture of symbols. The interesting point is that this degradation does not depend on SNR. In other words, the slopes of $\bar{P}_{e}$ curves, which signifies the order of diversity of the system, are almost the same for D2SD and SIMO (see (27) and (28)). ${ }^{8}$

To see the utility the analytical expressions, we have also shown in Fig.7 curves of $P_{u p}^{\prime}$ and its approximation in high SNR from (27). It is seen that $P_{u p}^{\prime}$ is quite useful and close to $P_{\text {upper }}$; however, its approximation (for high SNR) is loose for $E_{b} / N_{0}<10 \mathrm{~dB}$.

\footnotetext{
${ }^{7}$ We assume the conditions of uncorrelated fading on the antenna elements for the case of SIMO system.

${ }^{8}$ Notice that since here we consider the performance in terms of $\bar{E}_{b} / N_{0}$ at receiver, the corresponding curve for SIMO system can also be considered as to belong to a double-transmit-antennas system, performing MRC detection at receiver by using Alamouti coding, for example [21,22]. Notice also that D2SD with $\mu=1$ may be regarded as a transposition of the Alamouti code [21] to the case of a single-antenna system.
} 


\subsection{Ricean fading}

For Ricean fading channels, the received signal can be considered to be composed of two components; one from line-of-sight (LOS) and the other one from multipath reflections. The former component can be assumed to be almost deterministic and constant, whereas the latter is a randomly varying component. We consider the channel gain $\alpha_{\text {Rice }}$ as [23]:

$$
\alpha_{\text {Rice }}=\sqrt{\mathrm{RF}}+\sqrt{1-\mathrm{RF}} \alpha_{\text {Ray }}
$$

where $\alpha_{\text {Ray }}$ is a unit-variance circularly symmetric complex Gaussian random variable representing random channel variations. RF is the ratio of the power received from LOS to the total received power. The usually employed Ricean K-factor [24] is in fact equal to RF/(1-RF). The PDF of $\rho=|\alpha|$, for this case is given by:

$$
P(\rho)=\frac{2 \rho}{1-\mathrm{RF}} \exp \left(-\frac{\rho^{2}+\mathrm{RF}}{1-\mathrm{RF}}\right) I_{0}\left(2 \rho \frac{\sqrt{\mathrm{RF}}}{1-\mathrm{RF}}\right) \quad ; \quad \rho \geq 0
$$

where $I_{0}($.$) is the modified Bessel function of first kind and zero order. As we did for the case of Rayleigh$ fading, we replace erfc(.) in the expression of $P_{\text {upper }}$ by $e^{-x^{2}}$ and average it over the PDF of $\rho$. After some manipulations, we obtain the following analytical expression for the new upper bound $P_{u p}^{\prime}$, which is looser than $P_{\text {upper }}$.

$$
\begin{gathered}
P_{u p}^{\prime}=\frac{\sigma^{4} / 4}{\left[\sigma^{2}+(1-\mathrm{RF})\right]\left[\sigma^{2}+\mu^{2}(1-\mathrm{RF})\right]} \exp \left(-\frac{\mathrm{RF}}{\sigma^{2}+(1-\mathrm{RF})}-\frac{\mu^{2} \mathrm{RF}}{\sigma^{2}+\mu^{2}(1-\mathrm{RF})}\right)+ \\
\frac{\sigma^{4} / 4}{\left[\sigma^{2}+(1+\mu)^{2}(1-\mathrm{RF})\right]\left[\sigma^{2}+(1-\mu)^{2}(1-\mathrm{RF})\right]} \exp \left(-\frac{(1+\mu)^{2} \mathrm{RF}}{\sigma^{2}+(1+\mu)^{2}(1-\mathrm{RF})}-\frac{(1-\mu)^{2} \mathrm{RF}}{\sigma^{2}+(1-\mu)^{2}(1-\mathrm{RF})}\right)
\end{gathered}
$$

In high SNR this bound is simplified to the following.

$$
P_{u p}^{\prime} \approx \frac{1}{4}\left(\frac{E_{b}}{N_{0}}\right)^{-2}\left(\mu^{-2}+\left(1-\mu^{2}\right)^{-2}\right)\left(1+\mu^{2}\right)^{2} \exp \left(-\frac{2 \mathrm{RF}}{1-\mathrm{RF}}\right) \quad ; \quad \frac{E_{b}}{N_{0}} \gg 1
$$

Note that for $\mathrm{RF}=0$ (Rayleigh fading), we find the same expression of (27).

\subsubsection{Choice of mixture factor $\mu$}

We have shown in Fig.8 curves of upper and lower bounds on $\bar{P}_{e}$ versus $\mu$ for five values of RF and $\bar{E}_{b} / N_{0}=10 \mathrm{~dB} . \mathrm{RF}=100 \%$ represents the LOS channel. It is seen that to obtain the best fading reduction, $\mu$ should be increased for increased RF. If the LOS contribution is not too high $(\mathrm{RF}<90 \%) \mu_{o p t}$ is between 0.55 and 0.65 . For negligibly fading channels $(\mathrm{RF}>90 \%) \mu_{o p t}$ is close to 1 . As a matter of fact, with less signal fading, the MF matrix $\boldsymbol{G}$ will be "more orthogonal." So, the effect of the interference will be less important, and a larger mixture factor $\mu$ can be chosen. This is in accordance with the 


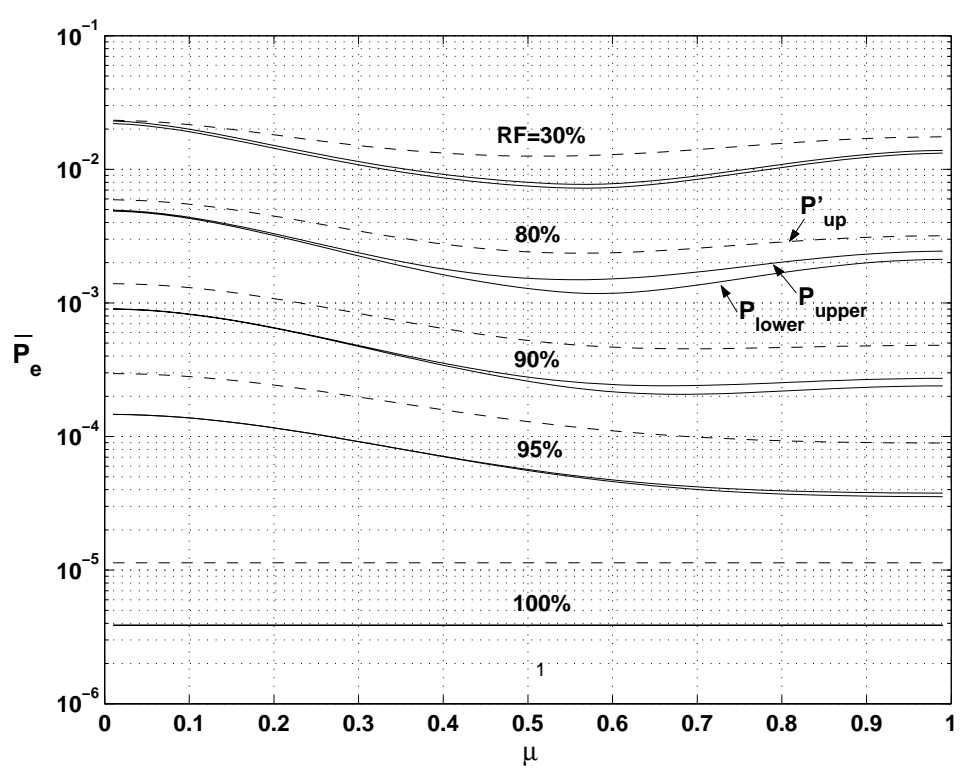

Figure 8: Choice of $\mu$, Ricean flat fading, $\bar{E}_{b} / N_{0}=10 \mathrm{~dB}$

results of Subsection 4.1: we can see from Fig.5 that for $f=2, \mu=1$ results in a better performance than $\mu=0$ or $\mu=0.5$. We have also shown in Fig.8 curves of $P_{u p}^{\prime}$. We see that this bound is quite useful as long as RF is not too large. The important difference between $P_{u p}^{\prime}$ and $P_{\text {upper }}$ for $\mathrm{RF}=100 \%$ is due to the difference of $\operatorname{erfc}(x)$ and $e^{-x^{2}}$.

\section{Discussion and conclusions}

For a simple single-antenna communication system D2SD with $\mu \geq 0.5$ provides an interesting gain in the system performance, whatever the channel statistics. This improvement is achieved at the cost of a negligible complexity and without any additional cost in the transmit power and with no loss in spectral efficiency. The choice of the imposed delay in signal transmission depends on the rapidity of channel variations. For this delay to be reasonable, D2SD is appropriate for relatively fast fading channels. Notice that this is also the case for the techniques of interleaved coding and modulation diversity. D2SD may not be considered as an alternative to channel coding. However, as compared to the case of interleaved coding, by D2SD we need much less redundancy to be added to data bits to obtain the same performance. On the other hand, D2SD is of considerable reduced complexity, as compared to concatenated codes.

In D2SD we focused on the diversity order of $L=2$ since by this choice we add a very negligible complexity to the system. Specially, this choice implies a delay of only $\tau_{c}$ in the signal transmission (emission/reception). For larger $L$ values, a better performance can be obtained, but the resulting system would not be suitable for a real-time or duplex signal transmission. As an example, for an indoor 
mobile radio application (which most often corresponds to the flat fading case [15]) with $f_{0}=5 \mathrm{GHz}$ and a speed of about $5 \mathrm{~km} / \mathrm{h}, \tau_{c}$ which is the imposed delay for $L=2$, is in the order of $40 \mathrm{~ms}$. In the following we contrast our work with the convolutive precoding and modulation diversity approaches.

\section{- Comparison with convolutive precoders}

D2SD is quite advantageous to the precoding techniques of $[4,5]$ regarding the complexity and the induced delay in signal transmission/detection. If for example, we compare the results of Fig.7 with those of [5], to obtain $\bar{P}_{e} \approx 10^{-4}$ at $\bar{E}_{b} / N_{0} \approx 20 \mathrm{~dB}$, the required precoder length precoder is about $20 \tau_{c}$. D2SD, however, needs a delay of $\tau_{c}$ only. Another advantage of D2SD is that despite convolutive precoders that require the noise variance in the fading equalizer section, it does not require the noise power for the detection of symbols. So, D2SD does not suffer from a mismatch in the noise power, as it may be the case for the convolutive precoders. Also, D2SD needs the channel gain in only two time instants for the detection of each pair of symbols, in contrast to much more time instants required by convolutive precoders for fading equalization. So, it should reasonably be much less sensitive to channel estimation errors.

\section{- Comparison with previous works on modulation diversity}

Although D2SD can be regarded as a special case of modulation diversity techniques, in this work we provided several interesting contributions. We provided the ML detector with a simple structure, which permits to detect the symbols separately. In contrast, the universal lattice decoder in [11], which is more general and more complex to implement, is based on the (joint) detection of vectors of symbols or in other words, the detection of "signal points". The work of [8], on the other hand, considers the bound on the cut-off rate of the ML detector, but treats only linear (suboptimal) detectors such as the MMSE detector. We also provided upper and lower bounds on the symbol error probability, and showed that they are tight enough, and hence, very useful for the performance analysis of the detector. We provided precisions on the choice of the mixture factor $\mu$ in different fading conditions including Ricean fading. In most of the references, only the classical case of Rayleigh fading is considered.

\section{References}

[1] W.C. Jakes, Microwave Mobile Communications, John Wiley \& Sons, New York, 1974. Reprinted by IEEE Press, 1998

[2] J.G. Proakis, Digital Communications, McGraw Hill, 3rd Ed., 1995

[3] M.A. Khalighi, K. Raoof, and G. Jourdain, "A Simple Prefiltering to Increase the Capacity of Fading Channels," Proc. Int. Conf. Telecom., ICT, June 2002, Beijing, China, Vol. 2, pp. 653-657 
[4] A. Wittenben, "An energy- and bandwith-efficient data transmission system for non-selective fading channels," in Proceedings of IEEE Globecom 1990, New York, NY, Vol.3, pp. 1968-1972

[5] G.W. Wornell, "Spread-response precoding for communication over fading channels," IEEE Transactions on Information Theory, vol.42, No.2, Mar. 1996, pp. 488-501

[6] H.V. Poor and G.W. Wornell, Wireless Communications, Signal Processing Perspectives, Prentice Hall Int., Inc., NJ, 1998

[7] K. Boullé and J.C. Belfiore, "Modulation schemes designed for the Rayleigh fading channel," Proceedings of CISS, Princeton, NJ, Mar. 1992.

[8] D. Rainish, "Diversity transform for fading channels," IEEE Transactions on Communications, Vol.44, No.12, Dec. 1996, pp. 1653-1661.

[9] X. Giraud and J.C. Belfiore, "Constellations matched to the Rayleigh fading channel," IEEE Transactions on Information Theory, Vol. 42, No. 1, Jan. 1996, pp. 106-115

[10] J. Boutros and E. Viterbo, "Signal space diversity: a power- and bandwidth-efficient diversity technique for the Rayleigh fading channel," IEEE Transactions on Information Theory, Vol.44, No. 4, July 1998, pp. 1453-1467.

[11] E. Viterbo and J. Boutros, "A universal lattice code decoder for fading channels," IEEE Transactions on Information Theory, Vol. 45, No. 5, July 1999, pp. 1639-1642

[12] G. Wade, Signal Coding and Processing, Cambridge Univ. Press, 2nd Ed., 1994

[13] D. Divsalar and M.K. Simon, "The design of trellis coded MPSK for fading channels: performance criteria," IEEE Transactions on Communications, Vol. 36, No.9, Sept.1988, pp. 1004-1012

[14] B.D. Jeličić and S. Roy, "Design of a trellis coded QAM for flat fading channels," IEEE Transactions on Vehicular Technology, Vol. 44, No. 1, Feb. 1995, pp. 192-201

[15] L. Ros, G. Jourdain, and M. Arndt, "Interpretation and performances of linear reception in downlink TD-CDMA and multi-sensor extension," Annals of Telecommunications, Vol. 56, No. 5/6, May-June 2001, pp. 275-290

[16] S. Verdù, Multiuser detection, Cambridge Univ. Press, 1998

[17] M.A. Khalighi, K. Raoof, and G. Jourdain, "Capacity of multi-antenna time-dispersive channels subject to fading," Proc. 18th GRETSI Conf., Sept. 2001, Toulouse, France, pp. 129-132

[18] A.M. Sayeed and B. Aazhang, "Joint multipath-doppler diversity in mobile wireless communications," IEEE Transactions on Communications, Vol. 47, No. 1, Jan. 1999, pp. 123-132

[19] H.L. Van Tress, Detection, Estimation and Modulation Theory, Part I, Wiley, NY, 1968.

[20] L. Ros and M.A. Khalighi, D2SD: A Diversity technique to improve signal transmission in flat fading channels, Internal Report \#01/2004, LIS Laboratory, May 2004.

[21] S.M. Alamouti, "A simple transmit diversity technique for wireless communications," IEEE Journal on Selected Areas in Communications, Vol. 16, No. 8, Oct. 1998, pp. 1451-1458

[22] M.A. Khalighi, K. Raoof, and G. Jourdain, "Capacity of wireless communication systems employing antenna arrays, a tutorial study," Wireless Personal Communications, Vol. 23, No. 3, Dec. 2002, pp. 321-352 
[23] M.A. Khalighi, J.M. Brossier, G. Jourdain, and K. Raoof, "On capacity of Ricean MIMO channels," Proceedings of PIMRC Symposium, Sept.-Oct. 2001, San Diego, CA, Vol. A, pp. 150-154

[24] G.L. Stüber, Principles of Mobile Communication, Kluwer Academic Publishers, 1996

[25] M.A. Khalighi and L. Ros, "A clever combination of transmit symbols to reduce flat fading effect," Proceedings of International Symposium on Signal Processing and its Applications (ISSPA), June 2003, Paris, France, Vol.1, pp. 293-296 